\title{
Association of sleep duration and sleep quality with the physical, social, and emotional functioning among Australian adults
}

\section{Lallukka, Tea}

2018-04

Lallukka , T , Sivertsen, B , Kronholm , E , Bin , Y S , Overland, S \& Glozier , N 2018 , ' Association of sleep duration and sleep quality with the physical, social, and emotional functioning among Australian adults ' , Sleep health , vol. 4 , no. 2 , pp. 194-200 . https://doi.org/10.1016/j.sleh.2017

http://hdl.handle.net/10138/310980

https://doi.org/10.1016/j.sleh.2017.11.006

cc_by_nc_nd

acceptedVersion

Downloaded from Helda, University of Helsinki institutional repository.

This is an electronic reprint of the original article.

This reprint may differ from the original in pagination and typographic detail.

Please cite the original version. 


\section{Association of sleep duration and sleep quality with the physical, social, and emotional functioning among Australian adults}

Tea Lallukka, ${ }^{\mathrm{a}, \mathrm{b}, \mathrm{c}}$ Børge Sivertsen, ${ }^{\mathrm{d}, \mathrm{e}, \mathrm{f}}$ Erkki Kronholm, ${ }^{\mathrm{a}}$ Yu Sun Bin, ${ }^{\mathrm{c}}$ Simon $\emptyset$ verland ${ }^{\mathrm{f}, \mathrm{g}}$ Nick Glozier ${ }^{\mathrm{c}, \mathrm{h}}$

Running head: Sleep Health and Functioning

\section{To Sleep Health}

a Finnish Institute of Occupational Health, Helsinki, Finland

Postal address: P.O. Box 40, FIN-00251 Helsinki, Finland, tea.lallukka@ttl.fi

Postal address: Lemminkäisenkatu 14-18 B, Fl-20520; Turku, Finland, erkki.kronholm@ttl.fi

${ }^{b}$ Department of Public Health, University of Helsinki, Finland, tea.lallukka@helsinki.fi

Postal address: P.O. Box 20 (Tukholmankatu 8 B), FIN-00014 University of Helsinki, Finland,

tea.lallukka@helsinki.fi

'Sydney Medical School, University of Sydney, Australia, yusun.bin@sydney.edu.au

Postal address: Sleep Group, D17 - Charles Perkins Centre, University of Sydney NSW Australia 2006

${ }^{d}$ The Regional Centre for Child and Youth Mental Health and Child Welfare, Uni Research Health

Postal address: Postboks 7810, 5020 Bergen, Norway

e Department of Research and Innovation, Helse-Fonna HF Haugesund Hospital

Postal address: Postbox 2170, 5504, Haugesund, Norway

f Department of Health Promotion, Norwegian Institute of Public Health, borge.sivertsen@fhi.no,

simon.overland@fhi.no

Postal address: Zander Kaaesgate 7, 5015 Bergen, Norway

${ }^{\mathrm{g}}$ Department of Psychosocial Science, University of Bergen, Norway

Postal address: Postboks 7807, 5020 Bergen, Norway

${ }^{\mathrm{h}}$ Brain and Mind Centre, University of Sydney, Sydney, NSW, Australia, nick.glozier@sydney.edu.au

Postal address: Brain and Mind Centre, 94 Mallett Street, Camperdown, NSW 2050

Word counts: Main text 4181 words, abstract 208 words \& 3 Tables, 4 Online Appendices

*Corresponding author

Tea Lallukka, PhD

Finnish Institute of Occupational Health

P.O. Box 18, FIN-00032 Helsinki, Finland

GSM: +35843825 4882, E-mail: tea.lallukka@ttl.fi 


\begin{abstract}
Objectives: We aimed to evaluate the interaction of two key determinants of sleep health, quantity and quality, with physical, emotional, and social functioning, in the general population.
\end{abstract}

Design: Nationally representative Australian cross-sectional study.

Setting: General population.

Participants: 14571 people aged 15 or older in Household, Income and Labour Dynamics in Australia (HILDA) in 2013.

Measurements: The associations of sleep quality (good/poor) in combination with midrange (6-8 hours), short $(<6)$ or long $(>8)$ sleep duration with functioning, determined from the SF-36, were evaluated using logistic regression adjusting for sociodemographic, relationships, health behaviours, obesity, pain, and mental and physical illness confounders.

Results: After adjusting for gender, and age, poor sleep quality in combination with short, mid-range and long sleep was associated with worse physical, emotional and social functioning. Pain and comorbid illness explained much of these associations, while attenuation from other covariates was minor. The associations of poor sleep quality with worse functioning remained after full adjustment regardless of sleep duration, while among people with good quality sleep, only those with long sleep duration reported poorer functioning.

Conclusions: Poor sleep quality has robust associations with worse functioning regardless of total duration in the general population. There appears to be a substantial number of functional short sleepers with good quality sleep.

Keywords: population-based; insomnia symptoms; sleep quantity; Australia; SF-36 


\section{Introduction}

Sleep health can be conceptualised as having several different dimensions e.g the five proposed by Buysse (1) and each may have different effects upon human function. The associations between sleep duration and health, work ability, and mortality have been studied in many different cohorts, with the results providing strong and relatively consistent evidence that short and long sleep duration increase the risk of adverse health outcomes $(2,3)$. Other studies have focused on sleep quality, typically characterised as insomnia, with similar consistency of evidence for its impact on health $(4,5)$. Although sleep duration (both objectively measured and subjectively reported) shows high inter-individual variability the vast majority of public health messages focus on everyone achieving a certain number of hours of sleep (6), only recently have these messages attempted to capture the risks related to poor sleep quality (7).

With few exceptions most of the studies have not considered the interaction of sleep duration and quality contributing to health functioning. Where assessed together, sleep quality and quantity have been jointly associated with ill-health, increased work disability and deficits in neuropsychological and psychomotor performance, with the most pronounced risks typically found among people with poor sleep quality and short sleep (8-14). In such studies on sleep quality and sleep duration, mid-range and long sleep durations have often been combined $(8,14)$. This is somewhat surprising, as even the first study published on the association between sleep duration and mortality showed that the risks are highest in both extreme ends of sleep duration distribution, i.e., among short and long sleepers (2). Moreover, both short and long sleepers report poorer sleep quality which suggests that each of these groups could be distinguished from people with sleep duration within population mean range $(15,16)$. Findings from The Penn State Cohort suggest for instance that understanding the impact of sleep disturbance on cognition requires knowledge and consideration of both dimensions simultaneously (14), but that study also combined mid-range and long sleep duration. Combining mid-range 
and long sleep assumes a linear correlation between sleep duration and health, despite consistent evidence that the association is actually curvilinear or $U$-shaped $(17,18)$.

To address the above gaps, we aimed to examine how combinations of sleep quality and sleep duration are associated with different dimensions of health functioning. The associations were examined in a population-based nationally representative Australian dataset with the ability to address potential confounders behind any such relationship such as pain, sociodemographic factors, health behaviours, and health. Based on the previous literature, it was expected that both poor sleep quality and short and long sleep duration contribute to poor health functioning, and that the most dysfunctional groups are people with poor sleep quality in combination with short or long sleep. 


\section{Participants and methods}

Data

Data for this study were derived from the ongoing Household, Income and Labour Dynamics in Australia (HILDA) panel survey $(19,20)$. The primary aims of the survey are to explore the dynamics of family and households, income and welfare and the labour market and as such respondents are less aware of health-related analyses. The data are nationally representative, with the initial reference population for wave 1 being all residents of Australia living in private dwellings (20). A multi-stage sampling approach was applied. Altogether 7,682 households and 19,914 individuals participated at wave 1 . The response rate at baseline was $66 \%$. In $2011,2,153$ households, and 5,451 persons were added to the cohort (response rate 69\%).

Cross-sectional data from wave 13 collected in 2013 were used for this study $(n=17,501)$, as sleep questions were first introduced in that survey. More than $95 \%$ of previous-wave respondents participated at wave 13 (19). We excluded those who died in 2013 and $2014(n=90)$ to reduce the effects of severe illnesses on reports of sleep and functioning (near death approach), leaving 14,557 participants, who provided data for the variables of interest, i.e., sleep, functioning and covariates.

\section{Sleep}

Sleep duration was self-reported in hours. It was asked separately from those currently employed and not employed. Both groups were also asked to separately report their sleep for weekdays/work day nights and weekends/non-work nights. From the responses, a sum variable of total sleep duration per week was computed, and this was divided by seven to get mean daily sleep duration for each participant, as we could not assume a 5:2 work: weekend split. The questions specifically asked about "How many hours of actual sleep do you usually get?" (on a work day night/ non-work night, weekdays and weekends), and are assumed to reflect actual sleep duration and not time 
in bed. Daily mean sleep duration was classified into short sleep (less than 6 hours per night), mid-range sleep ( 6 to 8 hours per night) and long sleep (more than 8 hours per night), mid-range broadly reflecting current adult recommendations (21). A more detailed classification was not feasible due to low numbers in the extreme ends of sleep duration combined with sleep quality.

Self-reported sleep quality was based on two core insomnia symptoms reflecting difficulties initiating or maintaining sleep (DIMS): reporting "trouble sleeping because cannot get to sleep within 30 minutes" and "trouble sleeping because wake up in the middle of the night or early in the morning", in addition to overall rating of sleep quality, reflecting daytime consequence of these insomnia symptoms. Five response alternatives ranged from "not during the past month" to "at least five times a week during past month" for insomnia symptoms, and from "very good" to "very bad" for overall sleep quality. Participants were considered to have poor sleep quality if they reported either difficulty initiating OR maintaining sleep at least three times or more per week during the past month AND rated their overall sleep quality as either "fairly bad" or "very bad". A categorical variable was then made classifying people into good or poor sleep quality and one of the three sleep duration categories, forming six groups (categories in the Appendix 1 and Table 2). Those with good quality and mid-range duration sleep served as a reference group.

\section{Covariates}

Covariates were all self-reported and comprised key sociodemographic factors, health behaviours, obesity, pain, physical and mental illnesses. These variables were identified a priori due to their established associations with sleep and functioning in these data and elsewhere (22-26). For the descriptive analyses, age was classified into two groups: 15 to 44 years, and 45 years or more, as poor sleep quality tends to increase particularly in midlife $(23,27)$ alongside declines in health and functioning $(26)$. In the multivariable 
analyses, we included age as a continuous variable. Employment status was dichotomized into those working (all current employees, employers or those selfemployed, and employees of their own business and $<1 \%$ who were unpaid family workers) and not working. Descriptive analysis was stratified by employment status, and the dummy variable was adjusted for in all the models, as sleep duration and sleep quality vary by employment status (23). This also better allows comparisons for previous studies that have mainly included employed people and have focused on work disability $(8,10,11)$. Marital status was defined as being married/de facto, with those separated, divorced, widowed or single combined to another category. Education was classified into three hierarchical groups: high (masters or doctorate, graduate diploma, graduate certificate, bachelor or honours), intermediate (advanced diploma, diploma, Certificate III or IV, completed high school) and low (less than high school). We also considered whether the participant was living with children aged 0-4 years (no/yes), as this might affect sleep $(19,23)$. Social connections were defined based on how often participants reported they "got together socially with friends or relatives that were not living with them". Seven response alternatives ranged from "every day" to "less often than once every 3 months".

Smoking was classified into three groups: never smokers, ex-smokers and current smokers. Alcohol use was defined similarly based on current and former drinking patterns: never drinkers, former drinkers, drinking alcohol 1-2 times a week or less, and drinking alcohol 3 times a week or more often. Body mass index (BMI) was based on self-reported weight and height $\left(\mathrm{kg} / \mathrm{m}^{2}\right)$, with participants divided into normal weight and obese $(\mathrm{BMI}>30)$ categories. Pain was based on responses to the SF-36 question about bodily pain in last 4 weeks with seven response alternatives ranging from "no bodily pain" to "very severe pain". Any chronic disease included any of depression or other mental illness, type I or II diabetes, heart disease or circulatory disease, hypertension, asthma, cancer, arthritis or osteoporosis or other serious illness. 
Health functioning

We used transformed scales of the role physical, role emotional and social functioning subscales of the SF-36 for the outcomes (28). The range of each of the transformed scores varied from 0 to 100 , with higher scores indicating better functioning. The validity of the SF-36 as well as the item-internal consistency, item-discriminant validity, and scale reliabilities in these data have been found to be good and subscales included in this study were shown to have sound psychometric properties, namely good internal consistency, discriminant validity and high reliability (28). We dichotomized each subscale to focus on the poorest functioning quartile, as has been commonly done in previous literature (29-31).

For sensitivity analyses we ran the same models classifying participants with any positive responses to the original items as having 'poor functioning', separately for all dimensions. This produced very similar distributions and associations, and we present the conventional transformed scales for final analyses only.

\section{Statistical analyses}

The descriptive results reflect characteristics of the HILDA wave 13 sample. We first described the distribution of sleep health and potential confounders in the study population by employment status (Table 1 ), and then cross-tabulated the categorical sleep exposure with each dimension of functioning (Table 2: results of chi-square tests are displayed). Cross-tabulations between the sleep exposure groups and covariates can be found in Appendix 1. Finally, separate logistic regression models were fitted (Table 3). Model 1 was adjusted for gender, age, and employment status, while model 2 additionally included all other sociodemographic and socioeconomic factors. Model 3 was adjusted for gender, age, health behaviours and obesity, model 4 for gender, age and social connections, model 5 for gender, age and pain. In model 6, we estimated the associations adjusting for all covariates simultaneously. As a sensitivity analysis, Appendix 2 shows that a finer categorisation of sleep duration does not substantially 
change the associations with health functioning. In further sensitivity analyses (Appendices 3-4), the impact of sleep duration and insomnia on functioning were modelled separately and the pattern of associations was as expected given the main results. All the analyses were conducted using an SAS Statistical Software, version 9.4 (SAS, Cary, NC, USA). 


\section{Results}

The sample characteristics are shown in Table 1, stratified by employment status. Roughly two thirds of all participants were employed. Participants not working were less educated, and more likely to have a chronic disease. Short sleep duration was more prevalent $(p<0.001)$ among those not employed $(23 \%)$ than those employed $(16 \%)$. The prevalence of long sleep was $27 \%$ among people not employed and $19 \%$ among those employed (Table 1). Poor sleep quality was also more common among people not employed than employed people ( $24 \%$ vs $17 \%)$. Distribution of the 6-category sleep duration/ sleep quality predictor is in Table 2, while the Online Appendix 1 displays the covariates association with sleep health.

Table 2 shows the prevalence of role limitations due to poor physical and emotional health and social functioning in different categories of sleep duration and quality. Participants with poor sleep quality were consistently more likely than those with good sleep quality to report poorer functioning in all three dimensions. Similarly, those with short and long sleep durations had poorer functioning than those reporting 6-8 hours of sleep, regardless of sleep quality. These patterns were similar for those employed and not employed, although the absolute level of poorer functioning was higher among people not employed.

The associations between sleep quantity and quality with health functioning were further examined using logistic regression (Table 3). After adjusting for gender, age, and employment status, all categories of sleep quality/quantity were associated with poorer functioning compared to mid-range sleep duration (6-8 hours) and good sleep quality. For the role physical subscale, the strongest associations were found for people reporting poor sleep quality, and short (OR 3.15; 95\% CI 2.76-3.61), mid-range (OR 3.00; 95\% CI 2.60-3.46) or long sleep (OR 4.60; 95\% CI 3.52-6.02). Adjustments for sociodemographic factors, employment, health behaviours and obesity made but a minor contribution to these associations, whereas somatic and mental illnesses and the level of 
pain reduced the associations the most. However, the association between sleep and physical functioning remained statistically significant, except for short sleep with good quality after full adjustment.

The association of sleep categories with the role emotional subscale largely followed those for physical subscale, with people reporting both poor sleep quality and short (OR $3.14 ; 95 \%$ CI $2.75-3.58$ ), mid-range (OR 3.32; 95\% CI 2.93-3.76) or long sleep (OR $4.75 ; 95 \%$ CI 3.69-6.11) being the most 'dysfunctional' groups. The effects of adjustment on the associations also followed those for role physical subscale. Thus, the associations similarly remained in the fully adjusted models, with the exception for participants with short duration but good quality sleep.

Finally, strong associations were also found for social functioning. The strongest associations were again found for people with poor sleep quality, in combination with short (OR 4.15; 95\% CI 3.65-4.72), mid-range (OR 3.26; 95\% CI 2.85-3.74) and long sleep (OR 6.60; 95\% CI 5.11-8.54). Adjusting for covariates followed a similar pattern as compared to the other forms of functioning.

Sensitivity analyses stratifying by employment status were conducted but as similar associations were observed, only the results of analyses adjusting for employment status are shown. Additionally, working hours were adjusted for in a sensitivity analysis among employed people to cover the potential effects of overtime on the associations, however, working hours (both continuous and categorized) made a negligible contribution to the examined associations. 


\section{Discussion}

In this population representative sample self-reported sleep quality and sleep duration interact to impact on physical, emotional, and role functioning such that good quality sleep of short duration is not associated with poor functioning. Poor sleep quality is strongly associated with worse daytime functioning regardless of duration whilst the association of long sleep duration on poorer function appears independent from that of sleep quality. These associations between poor sleep quality and functioning remained significant, if attenuated in size, after considering a priori factors considered likely confounders. People with poor sleep quality had worse functioning even in those with mid-range sleep durations.

\section{Interpretation and implications}

Previous studies on the interaction of sleep duration and quality have not considered different dimensions of functioning. Thus, the novelty of this study is that the association between sleep quality and functioning holds across all categories of sleep duration and three domains of function. While our results are in line with previous studies focusing on sleep quantity and quality as separate factors, and their association with quality of life (32-34), comparability to this study remains limited, as these previous studies did not address sleep duration and sleep quality simultaneously. Quality of life has also been measured in various ways from single reports about perceived quality of life using the SF-36 survey. For instance, previous studies have reported that poor sleep quality is associated with poorer emotional reactions, social isolation and physical mobility (35), and lower scores on all dimensions of health functioning $(36,37)$.

Being circumspect that our study is cross-sectional, focus on both sleep quality and duration simultaneously is of importance, as short sleep of good quality appeared to have no or but weak associations with functioning. Furthermore, there is very little or no difference between short and mid-range sleep duration groups in the presence of poor sleep, which is a novel finding and could be further elaborated in prospective studies of 
different health outcomes. In previous studies focusing on sleep duration only (34), it is thus possible that the adverse effects have mainly been related to poor sleep quality and not the duration of sleep.

Accordingly, we found that poor sleep quality combined with any sleep duration was associated with both physical and emotional function, with little or no difference between mid-range and short sleepers. This is in contrast to other studies which suggest that insomnia with mid-range sleep duration is associated with sleep misperception and cognitive-emotional arousal, and that only insomnia with short sleep is linked to worse mental and physical health (13). However, we used self-reported sleep duration in the current study rather than polysomnographic determination of sleep time, so it is possible that objectively measured short sleep has distinct effects on functioning.

Due to a relatively large variation in sleep duration in populations $(23,34)$, for some people 'short' or 'long' sleep by our definition could reflect their preferred sleep pattern. It is assumed that functioning is normal when sleep duration meets the perceived need for sleep. This assumption is supported by our results showing little difference in functioning for people with different sleep durations and good sleep quality, particularly after considering all potential confounders. One might expect that if sleep duration is very extreme (short or long), adverse health effects could emerge (21) but based on our findings, this does not appear to be case for people with short habitual sleep, independent of pre-existing health conditions. As having poor sleep quality is likely worse for functioning than extreme sleep durations, these results suggest that a public health message might need to shift from asking "did you get enough sleep" to "did you sleep well".

With respect to poor sleep quality and long sleep, one explanation for the strong associations with poor functioning could be assumed to be linked to pre-existing illhealth. However, as we omitted all deaths occurring during the year of participation and 
the year after, and as we controlled for various serious mental and physical illnesses, this explanation does not seem plausible.

The group of people with long and poor quality sleep is small, and hence of limited public health relevance, but further studies are warranted to better understand who reports long sleep in combination with poor quality and why. In the current study population, the characteristics of people with long and poor quality sleep do not appear dramatically different to other people with poor quality sleep (Appendix 1) with similar sociodemographic background, health behaviours, obesity and health characteristics. As the U-shaped association between sleep duration and reports of sleep problems is wellestablished $(15,16)$, future studies could corroborate which factors account for varying sleep duration among people with poor quality sleep, particularly with objective indicators of sleep quality such as sleep efficiency and sleep fragmentation. Based on the current results, it appears that perceived sleep quality is more important than sleep duration for functioning.

The approach chosen to examine the contribution of sleep quality and quantity to health functioning could also be of importance. For example, person-orientated methods such as latent class analyses and also cluster analyses have been used in some studies to find what kind of groups naturally occur and exist in the populations (38-40). To examine, if more information could be revealed using a person-orientated approach, we initially also used latent class analysis. Those analyses also supported that sleep duration deviant from the population mean combined with poor sleep quality, is most detrimental to health functioning (data not shown). Producing similar findings with different methods provides more compelling evidence about the significance of sleep quality to health, independent of sleep duration.

Finally, some previous studies have also questioned the implications of short and long sleep. For example, previous studies have not found an association between sleep duration and mortality in midlife (41), or between sleep duration and CRP (42). 
Background mechanisms of long sleep - health association have also been discussed and problematized (43).

Methodological considerations

When further interpreting our results, it needs to be acknowledged that self-reported data on sleep duration and poor sleep quality could have led to some bias. Sleep duration is known to be overestimated compared to objective measures, especially among people with short sleep $(44,45)$. In turn, insomniacs with objective normal sleep duration have been shown underestimate their sleep (46). As the actual prevalence of short or long sleep or poor quality sleep was not the focus of the study, we cannot see whether this bias should be associated with our functional outcomes. Negative reporting bias could be assumed to affect the results, if those reporting poorest sleep also were more likely to report worse function. However, with regards to sleep duration, short or long sleep is not by definition negative, as e.g. people with short sleep do not necessarily have worse functioning. To our knowledge, there are no studies focusing on objective sleep duration and sleep quality and functioning, and thus it is difficult to confirm the actual contribution of negative reporting bias to our results. Still, even self-reported symptoms such as insufficient or poor quality sleep should not be ignored, and studies of their associations with different health outcomes such as functioning, and elaboration of mechanisms that explain the associations are needed.

The included sleep quality items are not fully similar to the recent recommendations (7), which we acknowledge as a limitation. However, in large epidemiological studies such as this one, it is not usually feasible to include lengthy multi-item measures. While crude, these included items, nonetheless, reflect core insomnia symptoms and should distinguish between poor and good sleepers. Moreover, the associations between individual insomnia symptoms and work disability have been very similar for both difficulties maintaining and initiating sleep (47). In future studies, more detailed measurements could help distinguish what types of symptoms contribute most strongly 
to the perception of sleep quality (e.g. initiation or maintenance). Nonetheless, due to the broad definition of sleep quality, we acknowledge that random misclassification leads to error that will most likely underestimate a true association.

It further needs to be acknowledged that sleep variables were introduced rather late in the HILDA cohort. However, the response rates have remained high among those who initially participated in the study, suggesting that health-related attrition is unlikely to distort the findings $(19,20)$. Nonetheless, if healthier people were more likely to participate at this follow-up survey, their sleep and functioning are assumed better as compared to total population. This makes our findings conservative. As the study was cross-sectional, reverse causality cannot be ruled out. This means that poorer sleep could lead to poorer functioning, or people with poor functioning could have shorter and poorer quality sleep.

Although self-reported, our measures of functioning have been well-validated, also in this population (28). However, it has already been previously proposed that the included scales are likely unable to capture the multifactorial nature of interference of insomnia to the dimensions of functioning such as social functioning (32). More detailed and in-depth effects could be better addressed in qualitative or intervention studies, targeted to the high risk groups such as the long sleepers with poor sleep quality identified in the present study. In other words, although some aspects of functional limitation are missed in questionnaire surveys, using validated questionnaires is, nonetheless, likely to capture at least in a proxy way the true phenomena about the contribution of sleep quantity and quality to the different dimensions of functioning.

A strength of this study is the inclusion of key correlates of sleep and functioning, and the ability to control for both physical and mental illnesses, pain, health behaviours and sociodemographic factors as well as social connections. This helped produce more robust evidence about the relative contribution of sleep quantity and quality to health 
functioning. A further strength is the inclusion and comparison of different dimensions of functioning, namely physical, emotional and social. Most studies have been focusing on mental health, and links between sleep and mental health (48), with social functioning receiving less attention. The apparently strong association and mechanisms between poor sleep and poor social functioning need to be further elaborated and corroborated using prospective data and more objective outcomes. As the associations are clear for different forms of functioning, this highlights the importance of focusing on wide domains of functioning. A further strength of this study is the opportunity to examine nationally representative Australian data comprising a sample of both those currently employed and not employed, and to show that the associations are not unique to working populations most often studied when focusing on the interaction between sleep quantity and quality $(8,10,11)$. Finally, as the initial focus of the study was not related to sleep or health, the participants' responses are less likely to have been affected or biased with respect to the current aims.

\section{Conclusions}

This study showed consistent associations between most combinations of poor sleep quantity and quality with functioning in a large population, representative of Australian adults. In line with previous work, poor sleep quality tends to dominate these associations, irrespective of sleep duration. In this general population short sleep in combination with good quality sleep does not appear to be associated with poorer functioning. These findings suggest that public health messages around sleep should focus less on achieving a "normal" sleep duration and more on achieving good quality sleep. 


\section{Funding}

TL was supported by the Academy of Finland [grant numbers \#287488, \#294096]. YSB was supported by a 2017 Career Development Award from the Sleep Research Society Foundation. Funding sources had no involvement in conducting the study

\section{Acknowledgements}

This paper uses unit record data from the Household, Income and Labour Dynamics in Australia (HILDA) Survey. The HILDA Project was initiated and is funded by the Australian Government Department of Social Services (DSS), and is managed by the Melbourne Institute of Applied Economic and Social Research (Melbourne Institute). The findings and views reported in this paper, however, are those of the author and should not be attributed to either DSS or the Melbourne Institute. 


\section{References}

(1) Buysse DJ. Sleep health: can we define it? Does it matter? Sleep 2014;37:9-17.

(2) Hammond EC. Some preliminary findings on physical complaints from a prospective study of 1,064,004 men and women. Am J Public Health Nations Health 1964;54:11-23.

(3) Cappuccio FP, Cooper D, D'Elia L, et al. Sleep duration predicts cardiovascular outcomes: a systematic review and meta-analysis of prospective studies. Eur Heart J $2011 ; 32: 1484-1492$.

(4) Sofi F, Cesari F, Casini A, et al. Insomnia and risk of cardiovascular disease: a metaanalysis. Eur J Prev Cardiol 2014;21:57-64.

(5) Li Y, Zhang X, Winkelman JW, et al. Association between insomnia symptoms and mortality: a prospective study of U.S. men. Circulation 2014;129:737-746.

(6) Glozier N. Maybe it's quality not length that matters. Sleep 2012;35:313-314.

(7) Ohayon M, Wickwire EM, Hirshkowitz M, et al. National Sleep Foundation's sleep quality recommendations: first report. Sleep Health 2017;3:6-19.

(8) Lian Y, Xiao J, Liu Y, et al. Associations between insomnia, sleep duration and poor work ability. J Psychosom Res 2015;78:45-51.

(9) Vgontzas AN, Fernandez-Mendoza J, Liao D, et al. Insomnia with objective short sleep duration: the most biologically severe phenotype of the disorder. Sleep Med Rev 2013;17:241-254.

(10) Lallukka T, Haaramo $P$, Rahkonen $O$, et al. Joint associations of sleep duration and insomnia symptoms with subsequent sickness absence: the Helsinki Health Study. Scand J Public Health 2013;41:516-523.

(11) Haaramo P, Rahkonen O, Lahelma E, et al. The joint association of sleep duration and sleep problems with disability retirement: A longitudinal register-linked study. Scand J Work Environ Health 2012;38:427-435.

(12) Fernandez-Mendoza J, Vgontzas AN, Liao D, et al. Insomnia with objective short sleep duration and incident hypertension: the Penn State Cohort. Hypertension 2012;60:929-935.

(13) Fernandez-Mendoza J, Vgontzas AN. Insomnia and its impact on physical and mental health. Curr Psychiatry Rep 2013;15:418.

(14) Fernandez-Mendoza J, Calhoun S, Bixler EO, et al. Insomnia with objective short sleep duration is associated with deficits in neuropsychological performance: a general population study. Sleep 2010;33:459-465.

(15) Grandner MA, Kripke DF. Self-reported sleep complaints with long and short sleep: a nationally representative sample. Psychosom Med 2004;66:239-241. 
(16) Park S, Cho MJ, Chang SM, et al. Relationships of sleep duration with sociodemographic and health-related factors, psychiatric disorders and sleep disturbances in a community sample of Korean adults. J Sleep Res 2010;19:567-577.

(17) Cappuccio FP, D'Elia L, Strazzullo P, et al. Sleep duration and all-cause mortality: a systematic review and meta-analysis of prospective studies. Sleep 2010;33:585-592.

(18) Kronholm E, Laatikainen T, Peltonen M, et al. Self-reported sleep duration, all-cause mortality, cardiovascular mortality and morbidity in Finland. Sleep Med 2011;12:215221.

(19) Wilkins R. The Household, Income and Labour Dynamics in Australia Survey: Selected Findings from Waves 1 to 14. The 11th Annual Statistical Report of the HILDA Survey. 2016:1-124.

(20) Wooden M, Watson N. The HILDA Survey and its Contribution to Economic and Social Research (So Far)'. Econ Rec 2007;83:208-231.

(21) Hirshkowitz M, Whiton K, Albert SM, et al. National Sleep Foundation's updated sleep duration recommendations: Final report. Sleep Health 2015;1:233-243.

(22) Kortt MA, Dollery B. Association between body mass index and health-related quality of life among an Australian sample. Clin Ther 2011;33:1466-1474.

(23) Lallukka T, Sares-Jäske L, Kronholm E, et al. Sociodemographic and socioeconomic differences in sleep duration and insomnia-related symptoms in Finnish adults. BMC Public Health 2012;12:565.

(24) Haario P, Rahkonen O, Laaksonen M, et al. Bidirectional associations between insomnia symptoms and unhealthy behaviours. J Sleep Res 2013;22:89-95.

(25) McNamee $P$, Mendolia $S$. The effect of chronic pain on life satisfaction: evidence from Australian data. Soc Sci Med 2014;121:65-73.

(26) Stenholm S, Westerlund $\mathrm{H}$, Head J, et al. Comorbidity and functional trajectories from midlife to old age: the Health and Retirement Study. J Gerontol A Biol Sci Med Sci $2015 ; 70: 332-338$.

(27) Sivertsen B, Krokstad S, Øverland S, et al. The epidemiology of insomnia: associations with physical and mental health. The HUNT-2 study. J Psychosom Res 2009;67:109-116.

(28) Butterworth P, Crosier T. The validity of the SF-36 in an Australian National Household Survey: demonstrating the applicability of the Household Income and Labour Dynamics in Australia (HILDA) Survey to examination of health inequalities. BMC Public Health $2004 ; 4: 44$.

(29) Hemingway $H$, Nicholson A, Stafford M, et al. The impact of socioeconomic status on health functioning as assessed by the SF-36 questionnaire: the Whitehall II Study. Am J Public Health 1997;87:1484-1490.

(30) Mäkinen T, Laaksonen M, Lahelma E, et al. Associations of childhood circumstances with physical and mental functioning in adulthood. Soc Sci Med 2006;62:1831-1839. 
(31) Lallukka T, Ferrie JE, Rahkonen O, et al. Change in economic difficulties and physical and mental functioning: Evidence from British and Finnish employee cohorts. Scand J Work Environ Health 2013;39:521-530.

(32) Kyle SD, Morgan K, Espie CA. Insomnia and health-related quality of life. Sleep Med Rev 2010;14:69-82.

(33) Ishak WW, Bagot K, Thomas S, et al. Quality of life in patients suffering from insomnia. Innov Clin Neurosci 2012;9:13-26.

(34) Magee CA, Caputi P, Iverson DC. Relationships between self-rated health, quality of life and sleep duration in middle aged and elderly Australians. Sleep Med 2011;12:346350.

(35) Bucquet D, Condon S, Ritchie K. The French version of the Nottingham Health Profile. A comparison of items weights with those of the source version. Soc Sci Med $1990 ; 30: 829-835$.

(36) Katz DA, McHorney CA. The relationship between insomnia and health-related quality of life in patients with chronic illness. J Fam Pract 2002;51:229-235.

(37) Léger D, Scheuermaier K, Philip P, et al. SF-36: evaluation of quality of life in severe and mild insomniacs compared with good sleepers. Psychosom Med 2001;63:4955.

(38) Buysse DJ, Hall ML, Strollo PJ, et al. Relationships between the Pittsburgh Sleep Quality Index (PSQI), Epworth Sleepiness Scale (ESS), and clinical/polysomnographic measures in a community sample. J Clin Sleep Med 2008;4:563-571.

(39) Tavernier R, Willoughby T. Are all evening-types doomed? Latent class analyses of perceived morningness-eveningness, sleep and psychosocial functioning among emerging adults. Chronobiol Int 2014;31:232-242.

(40) Wennman H, Kronholm E, Partonen T, et al. Interrelationships of Physical Activity and Sleep with Cardiovascular Risk Factors: a Person-Oriented Approach. Int J Behav Med 2015;22:735-747.

(41) Gangwisch JE, Heymsfield SB, Boden-Albala B, et al. Sleep duration associated with mortality in elderly, but not middle-aged, adults in a large US sample. Sleep 2008;31:1087-1096.

(42) Taheri S, Austin D, Lin L, et al. Correlates of serum C-reactive protein (CRP)--no association with sleep duration or sleep disordered breathing. Sleep 2007;30:991-996.

(43) Grandner MA, Drummond SP. Who are the long sleepers? Towards an understanding of the mortality relationship. Sleep Med Rev 2007;11:341-360.

(44) Lauderdale DS, Knutson KL, Yan LL, et al. Self-reported and measured sleep duration: how similar are they? Epidemiology 2008;19:838-845.

(45) Cespedes EM, Hu FB, Redline S, et al. Comparison of Self-Reported Sleep Duration With Actigraphy: Results From the Hispanic Community Health Study/Study of Latinos Sueno Ancillary Study. Am J Epidemiol 2016;183:561-573. 
(46) Fernandez-Mendoza J, Calhoun SL, Bixler EO, et al. Sleep misperception and chronic insomnia in the general population: role of objective sleep duration and psychological profiles. Psychosom Med 2011;73:88-97.

(47) Lallukka T, Haaramo $P$, Lahelma $E$, et al. Sleep problems and disability retirement: a register-based follow-up study. Am J Epidemiol 2011;173:871-881.

(48) Baglioni C, Battagliese G, Feige B, et al. Insomnia as a predictor of depression: A meta-analytic evaluation of longitudinal epidemiological studies. J Affect Disord 2011;135:10-19. 
Table 1. Distributions of study variables in HILDA wave 13 (column \%), stratified by employment

All $(n=14571) \quad$ Employed $(n=9272) \quad$ Not Employed $(n=5299)$

$\begin{array}{lll}\text { (col\%) } & \text { (col\%) } & \text { (col\%) }\end{array}$

Gender

Men

46.7

50.7

39.6

Women

53.3

49.3

60.5

Age group

15-44 years

51.2

59.6

36.5

45 years or more

48.8

40.4

63.5

Current employment status

Working

63.6

100

0

Not working

36.4

0

100

\section{Marital status}

Married/ cohabiting

64.3

68.4

57.1

Separated, divorced, widowed, single

35.7

31.6

42.9

\section{Education}

High

25.5

31.4

15.1

Intermediate

47.0

50.9

40.1

Low

27.6

17.7

44.9

Children aged 0-4 years in household

No

87.1

86.0

88.9

Yes

12.9

14.0

11.1

Body mass index

Normal weight

77.3

78.5

75.3

Obese $(B M I=>30)$

22.7

21.5

24.7

\section{Smoking}

Never smoker

54.9

56.7

51.7

Ex-smoker

27.4

26.1

29.8

Current smoker

17.7

17.3

18.5

Alcohol drinking

Never drinker

11.3

7.7

17.6 
Former drinker

Current drinker, 1-2 times a week or rarer

Current drinker, 3-7 times a week

Any chronic disease ${ }^{a}$

No

Yes

Poor sleep quality

No

Yes

Sleep duration

Less than 6 hours

6-8 hours

More than 8 hours
7.7

25.9

55.1

55.2

44.8

80.5

83.0

76.2

19.5

17.0

23.8

18.9

16.3

23.3

59.4

21.8
64.7

19.0
50.1

26.6

a Depression or other mental illness, diabetes type I or II, heart/circulatory disease, hypertension, asthma, cancer, arthritis or osteoporosis or other serious illness 
Table 2. Prevalence of outcome by sleep quality and quantity in HILDA wave 13 ( $n=14571$; row \%)

\begin{tabular}{|c|c|c|c|c|}
\hline & $\begin{array}{c}\text { Poor } \\
\text { physical } \\
\text { functioning } \\
\text { (row\%) }\end{array}$ & $\begin{array}{c}\text { Poor } \\
\text { emotional } \\
\text { functioning } \\
\text { (row\%) }\end{array}$ & $\begin{array}{c}\text { Poor social } \\
\text { functioning } \\
\text { (row\%) }\end{array}$ & $\begin{array}{c}\text { Total row } \\
\text { numbers (\%) }\end{array}$ \\
\hline \multicolumn{5}{|l|}{ All } \\
\hline \multicolumn{5}{|l|}{ Sleep quality and quantity } \\
\hline Good quality, 6-8 hours of sleep & 16.7 & 17.1 & 14.8 & $7437(51.0 \%)$ \\
\hline Good quality, short sleep & 23.4 & 21.3 & 21.2 & $1397(9.6 \%)$ \\
\hline Good quality, long sleep & 23.0 & 22.1 & 20.2 & $2901(19.9 \%)$ \\
\hline Poor quality, 6-8 hours of sleep & 34.7 & 39.9 & 36.4 & $1219(8.4 \%)$ \\
\hline Poor quality, short sleep & 42.2 & 43.7 & 45.6 & $1349(9.3 \%)$ \\
\hline Poor quality, long sleep & 44.8 & 52.6 & 56.0 & $268(1.8 \%)$ \\
\hline$p$-value (chi-squared test) & $<.0001$ & $<.0001$ & $<.0001$ & \\
\hline Total \%, N & 23.0 & 23.5 & 21.9 & $14571(100 \%)$ \\
\hline \multicolumn{5}{|l|}{ Employed } \\
\hline \multicolumn{5}{|l|}{ Sleep quality and quantity } \\
\hline Good quality, 6-8 hours of sleep & 10.8 & 14.2 & 11.2 & $5225(56.4 \%)$ \\
\hline Good quality, short sleep & 13.1 & 17.4 & 15.7 & $839(9.1 \%)$ \\
\hline Good quality, long sleep & 14.2 & 15.9 & 14.3 & $1633(17.6 \%)$ \\
\hline Poor quality, 6-8 hours of sleep & 24.5 & 33.9 & 27.1 & $776(8.4 \%)$ \\
\hline Poor quality, short sleep & 26.9 & 33.2 & 31.9 & $674(7.3 \%)$ \\
\hline Poor quality, long sleep & 29.6 & 46.4 & 42.4 & $125(1.4 \%)$ \\
\hline$p$-value (chi-squared test) & $<.0001$ & $<.0001$ & $<.0001$ & \\
\hline Total \%, N & 14.2 & 18.3 & 15.4 & $9272(100 \%)$ \\
\hline
\end{tabular}

Not employed

Sleep quality and quantity

Good quality, 6-8 hours of sleep

30.6

23.7

23.5

$2212(41.7 \%)$

Good quality, short sleep

38.9

27.2

29.6

$558(10.5 \%)$ 


\begin{tabular}{lcccc} 
Good quality, long sleep & 34.3 & 30.1 & 27.9 & $1268(23.9 \%)$ \\
Poor quality, 6-8 hours of sleep & 52.6 & 50.3 & 52.8 & $443(8.4 \%)$ \\
Poor quality, short sleep & 57.5 & 54.1 & 59.3 & $675(12.7 \%)$ \\
Poor quality, long sleep & 58.0 & 58.0 & 67.8 & $143(2.7 \%)$ \\
$\quad p$-value (chi-squared test) & $<.0001$ & $<.0001$ & $<.0001$ & $5299(100 \%)$ \\
Total \%, N & 38.4 & 32.6 & 33.4 & \\
\hline
\end{tabular}


Table 3. Associations of sleep quality and quantity with poor physical, emotional and social functioning in HILDA wave 13 ( $n=14571)$

\begin{tabular}{|c|c|c|c|c|c|c|c|c|c|c|c|c|}
\hline \multirow{5}{*}{ Sleep quality and duration } & \multicolumn{2}{|c|}{ Model 1: age } & \multicolumn{2}{|c|}{ Model 2: Model 1+ } & \multicolumn{2}{|c|}{ Model 3: Model 1+, } & \multicolumn{2}{|c|}{ Model 4: Model 1} & \multicolumn{2}{|c|}{ Model 5: Model } & \multicolumn{2}{|c|}{ Model 6: all covariates in } \\
\hline & & nder, and & mari & status, young & smo & ing, alcohol & & + social & & $1+$ pain & Mod & + any serious \\
\hline & \multicolumn{2}{|c|}{ employment status } & \multicolumn{2}{|c|}{ children, education, } & \multicolumn{2}{|c|}{ and body mass } & \multicolumn{2}{|c|}{ connections } & & & \multicolumn{2}{|c|}{ mental or physical health } \\
\hline & \multicolumn{2}{|c|}{ adjusted for } & \multicolumn{2}{|c|}{ employment status } & \multicolumn{2}{|r|}{ index } & \multicolumn{2}{|c|}{ adjusted for } & & & \multicolumn{2}{|c|}{ condition } \\
\hline & OR & $(95 \% \mathrm{Cl})$ & OR & $(95 \% \mathrm{Cl})$ & OR & $(95 \% \mathrm{Cl})$ & OR & $(95 \% \mathrm{Cl})$ & OR & $(95 \% \mathrm{Cl})$ & OR & $(95 \% \mathrm{Cl})$ \\
\hline \multicolumn{13}{|l|}{ Poor physical functioning } \\
\hline Good quality, 6-8 hours & 1.00 & & 1.00 & & 1.00 & & 1.00 & & 1.00 & & 1.00 & \\
\hline Good quality, short sleep & 1.25 & $(1.08-1.45)$ & 1.24 & $(1.07-1.44)$ & 1.20 & $(1.03-1.39)$ & 1.25 & $(1.08-1.45)$ & 0.96 & $(0.81-1.14)$ & 0.95 & $(0.80-1.12)$ \\
\hline Good quality, long sleep & 1.40 & $(1.25-1.57)$ & 1.38 & $(1.23-1.55)$ & 1.40 & $(1.24-1.57)$ & 1.41 & $(1.26-1.58)$ & 1.36 & $(1.19-1.55)$ & 1.34 & $(1.18-1.53)$ \\
\hline Poor quality, 6-8 hours & 3.00 & $(2.60-3.46)$ & 2.99 & $(2.59-3.45)$ & 2.85 & $(2.46-3.29)$ & 2.92 & $(2.53-3.36)$ & 1.82 & $(1.54-2.15)$ & 1.74 & $(1.47-2.06)$ \\
\hline Poor quality, short sleep & 3.15 & $(2.76-3.61)$ & 3.12 & $(2.72-3.56)$ & 2.88 & $(2.52-3.3)$ & 3.04 & $(2.66-3.48)$ & 1.49 & $(1.27-1.74)$ & 1.41 & $(1.20-1.65)$ \\
\hline Poor quality, long sleep & 4.60 & $(3.52-6.02)$ & 4.49 & $(3.44-5.88)$ & 4.33 & $(3.30-5.70)$ & 4.43 & $(3.38-5.81)$ & 2.50 & $(1.80-3.48)$ & 2.32 & $(1.67-3.24)$ \\
\hline \multicolumn{13}{|l|}{ Poor emotional } \\
\hline \multicolumn{13}{|l|}{ functioning } \\
\hline Good quality, 6-8 hours & 1.00 & & 1.00 & & 1.00 & & 1.00 & & 1.00 & & 1.00 & \\
\hline Good quality, short sleep & 1.22 & $(1.06-1.41)$ & 1.22 & $(1.06-1.41)$ & 1.18 & $(1.02-1.36)$ & 1.22 & $(1.05-1.4)$ & 1.10 & $(0.95-1.27)$ & 1.07 & $(0.92-1.24)$ \\
\hline Good quality, long sleep & 1.26 & $(1.13-1.40)$ & 1.22 & $(1.09-1.36)$ & 1.26 & $(1.13-1.41)$ & 1.27 & $(1.14-1.41)$ & 1.22 & $(1.09-1.36)$ & 1.19 & $(1.06-1.33)$ \\
\hline
\end{tabular}




\begin{tabular}{|c|c|c|c|c|c|c|c|c|c|c|c|c|}
\hline Poor quality, 6-8 hours & 3.14 & $(2.75-3.58)$ & 3.15 & $(2.76-3.59)$ & 2.99 & $(2.62-3.42)$ & 3.01 & $(2.64-3.44)$ & 2.46 & $(2.15-2.82)$ & 2.27 & $(1.97-2.61)$ \\
\hline Poor quality, short sleep & 3.32 & $(2.93-3.76)$ & 3.31 & $(2.91-3.75)$ & 3.07 & $(2.71-3.49)$ & 3.15 & $(2.78-3.58)$ & 2.34 & $(2.05-2.67)$ & 2.10 & $(1.83-2.41)$ \\
\hline Poor quality, long sleep & 4.75 & $(3.69-6.11)$ & 4.58 & $(3.56-5.90)$ & 4.57 & $(3.54-5.89)$ & 4.49 & $(3.48-5.78)$ & 3.51 & $(2.70-4.57)$ & 3.03 & $(2.31-3.97)$ \\
\hline \multicolumn{13}{|l|}{ Poor social functioning } \\
\hline Good quality, 6-8 hours & 1.00 & & 1.00 & & 1.00 & & 1.00 & & 1.00 & & 1.00 & \\
\hline Good quality, short sleep & 1.39 & $(1.20-1.61)$ & 1.38 & $(1.19-1.60)$ & 1.32 & $(1.14-1.53)$ & 1.38 & $(1.19-1.6)$ & 1.16 & $(0.99-1.36)$ & 1.11 & $(0.95-1.31)$ \\
\hline Good quality, long sleep & 1.31 & $(1.17-1.47)$ & 1.26 & $(1.13-1.42)$ & 1.31 & $(1.17-1.47)$ & 1.33 & $(1.18-1.49)$ & 1.24 & $(1.1-1.41)$ & 1.21 & $(1.07-1.37)$ \\
\hline Poor quality, 6-8 hours & 3.26 & $(2.85-3.74)$ & 3.26 & $(2.84-3.74)$ & 3.04 & $(2.65-3.49)$ & 3.09 & $(2.69-3.55)$ & 2.22 & $(1.91-2.58)$ & 2.00 & $(1.71-2.33)$ \\
\hline Poor quality, short sleep & 4.15 & $(3.65-4.72)$ & 4.09 & $(3.59-4.65)$ & 3.73 & $(3.28-4.25)$ & 3.89 & $(3.42-4.43)$ & 2.46 & $(2.13-2.83)$ & 2.15 & $(1.85-2.49)$ \\
\hline Poor quality, long sleep & 6.60 & $(5.11-8.54)$ & 6.31 & $(4.88-8.17)$ & 6.23 & $(4.80-8.09)$ & 6.19 & $(4.77-8.03)$ & 4.51 & $(3.37-6.03)$ & 3.81 & $(2.83-5.12)$ \\
\hline
\end{tabular}


Appendix 1. Study variables (column \%) by combinations of sleep quality and duration among participants in HILDA wave $2013(n=14571)$

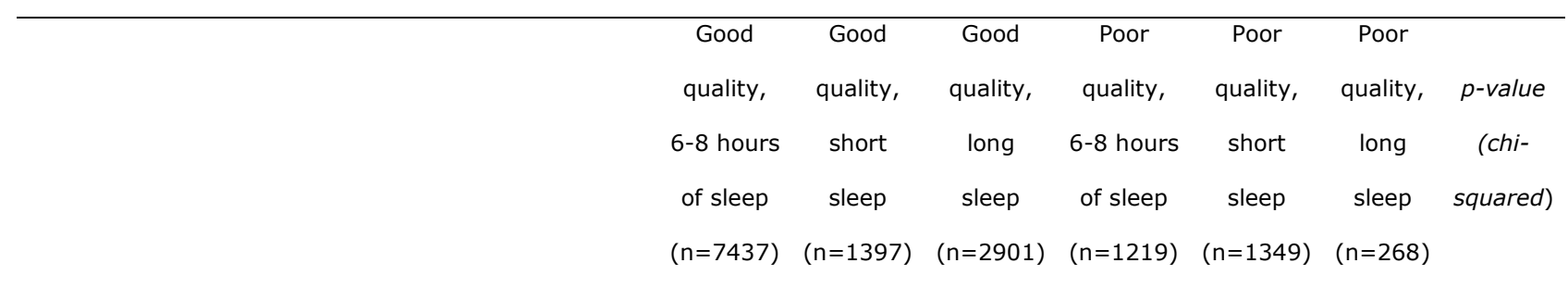

\section{Gender}

Men

Women

\section{Age group}

$15-44$ years

45 years or more

\section{Current employment status}

Employed

Not employed

\section{Marital status}

Married/ de facto

Single

\section{Education}

High

Intermediate

Low

\section{Children aged $0-4$ years}

No

Yes

\section{Body mass index}

Normal weight

Obese $(\mathrm{BMI}=>30()$

$\begin{array}{lllllll}49.3 & 48.8 & 45.2 & 42.4 & 38.6 & 39.6 & \\ 50.7 & 51.3 & 54.8 & 57.6 & 61.5 & 60.5 & <.0001\end{array}$

$\begin{array}{llllll}51.8 & 43.0 & 56.8 & 52.8 & 41.9 & 57.1\end{array}$

$\begin{array}{lllllll}48.2 & 57.1 & 43.2 & 47.3 & 58.1 & 42.9 & <.0001\end{array}$

$\begin{array}{llllll}70.3 & 60.1 & 56.3 & 63.7 & 50.0 & 46.6\end{array}$

$\begin{array}{lllllll}29.7 & 39.9 & 43.7 & 36.3 & 50.0 & 53.4 & <.0001\end{array}$

$\begin{array}{lllllll}67.9 & 65.6 & 56.9 & 63.3 & 62.3 & 53.0 & \\ 32.1 & 34.4 & 43.1 & 36.7 & 37.7 & 47.0 & <.0001\end{array}$

$\begin{array}{llllll}29.7 & 19.9 & 21.8 & 26.7 & 15.9 & 16.4\end{array}$

$\begin{array}{llllll}47.4 & 47.3 & 45.2 & 45.8 & 48.7 & 48.1\end{array}$

$\begin{array}{lllllll}22.9 & 32.8 & 33.0 & 27.5 & 35.3 & 35.5 & <.0001\end{array}$

$\begin{array}{llllll}86.7 & 82.4 & 91.9 & 85.2 & 84.4 & 93.3\end{array}$

$\begin{array}{lllllll}13.3 & 17.6 & 8.1 & 14.8 & 15.6 & 6.7 & <.0001\end{array}$

$\begin{array}{llllll}79.2 & 74.6 & 80.8 & 72.4 & 68.4 & 69.8\end{array}$

$\begin{array}{lllllll}20.8 & 25.4 & 19.2 & 27.7 & 31.6 & 30.2 & <.0001\end{array}$

\section{Smoking}

Never smoker

Ex-smoker

$\begin{array}{llllll}57.6 & 49.1 & 60.6 & 46.6 & 42.2 & 48.5 \\ 27.2 & 31.0 & 24.0 & 29.1 & 30.8 & 27.2\end{array}$


Current smoker

\section{Alcohol drinking}

Never drinker

Former drinker

Current drinker, 1-2 times a week or rarer

Current drinker, 3-7 times a week

\section{Current pain}

No

Intermediate

Severe

Meeting with friends/relatives

Every day, several times a week

About once a week, 2-3 times a month

About once a month or less

Any chronic disease ${ }^{a}$

No

Yes

a Depression or other mental illness, diabetes type I or II, heart/circulatory disease, hypertension, asthma, cancer, arthritis or osteoporosis or other serious illness

$\begin{array}{lllllll}9.9 & 12.4 & 16.3 & 8.4 & 9.3 & 12.3 & \\ 6.3 & 7.9 & 7.9 & 9.8 & 11.9 & 14.2 & \\ 27.1 & 26.4 & 22.5 & 27.0 & 25.0 & 25.4 & \\ 56.6 & 53.3 & 53.3 & 54.8 & 53.8 & 48.1 & <.0001\end{array}$

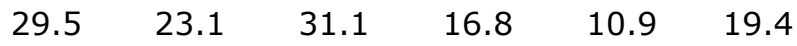

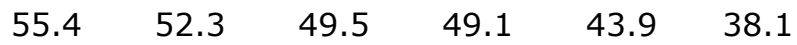

$\begin{array}{lllllll}15.1 & 24.6 & 19.4 & 34.0 & 45.2 & 42.5 & <.0001\end{array}$

$\begin{array}{llllll}28.0 & 28.6 & 33.9 & 23.9 & 21.7 & 22.8\end{array}$

$\begin{array}{llllll}53.1 & 48.4 & 48.5 & 49.2 & 49.2 & 48.9\end{array}$

$\begin{array}{lllllll}18.9 & 23.0 & 17.6 & 26.9 & 29.1 & 28.4 & <.0001\end{array}$

$\begin{array}{llllll}60.4 & 51.5 & 56.6 & 47.7 & 37.2 & 39.2\end{array}$

$\begin{array}{lllllll}39.6 & 48.5 & 43.4 & 52.3 & 62.8 & 60.8 & <.0001\end{array}$ 
Appendix 2. Prevalence of outcome by sleep quality and detailed quantity in HILDA wave $13(n=14571)$

\begin{tabular}{|c|c|c|c|c|}
\hline Sleep quality and duration & $\begin{array}{c}\text { Poor } \\
\text { physical } \\
\text { functioning } \\
\text { (row \%) }\end{array}$ & $\begin{array}{c}\text { Poor } \\
\text { emotional } \\
\text { functioning } \\
\text { (row \%) }\end{array}$ & $\begin{array}{c}\text { Poor social } \\
\text { functioning } \\
\text { (row \%) }\end{array}$ & $\begin{array}{c}\text { Total row } \\
\text { numbers (\%) }\end{array}$ \\
\hline Good quality, 6-8 hours of sleep & 16.7 & 17.1 & 14.8 & $7437(51.0 \%)$ \\
\hline Good quality, very short sleep (less than 5 hours) & 26.0 & 22.2 & 26.0 & $316(2.2 \%)$ \\
\hline Good quality, short sleep ( 5 hours to less than 6 hours) & 22.7 & 21.1 & 19.9 & $1081(7.4 \%)$ \\
\hline Good quality, longish sleep (more than 8 hours to less than 9 hours) & 19.9 & 19.9 & 17.0 & $1982(13.6 \%)$ \\
\hline Good quality, long sleep ( 9 hours to less than 10 hours) & 24.4 & 22.5 & 22.2 & $614(4.2 \%)$ \\
\hline Good quality, very long sleep (10 hours or more) & 39.7 & 35.7 & 37.4 & $305(2.1 \%)$ \\
\hline Poor quality, 6-8 hours of sleep & 34.7 & 39.9 & 36.4 & $1219(8.4 \%)$ \\
\hline Poor quality, very short sleep (less than 5 hours) & 47.0 & 48.2 & 50.2 & $662(4.5 \%)$ \\
\hline Poor quality, short sleep ( 5 hours to less than 6 hours) & 37.6 & 39.3 & 41.2 & $687(4.7 \%)$ \\
\hline Poor quality, longish sleep (more than 8 hours to less than 9 hours) & 37.7 & 47.9 & 49.1 & $167(1.2 \%)$ \\
\hline Poor quality, long sleep ( 9 hours to less than 10 hours) & 56.7 & 58.3 & 66.7 & $60(0.4 \%)$ \\
\hline Poor quality, very long sleep (10 hours or more) & 56.1 & 63.4 & 68.3 & $41(0.3 \%)$ \\
\hline$p$-value & $<.0001$ & $<.0001$ & $<.0001$ & \\
\hline Total \%, N & 23.0 & 23.5 & 21.9 & $14571(100 \%)$ \\
\hline
\end{tabular}


Appendix 3. Associations of sleep quantity with physical, emotional and social functioning in HILDA wave 13 ( $n=14571$ )

\begin{tabular}{|c|c|c|c|c|c|c|c|c|c|c|c|c|}
\hline \multirow{2}{*}{ Sleep duration } & \multicolumn{2}{|c|}{$\begin{array}{c}\text { Model 1: age, gender, } \\
\text { and employment status } \\
\text { adjusted for }\end{array}$} & \multicolumn{2}{|c|}{$\begin{array}{l}\text { Model 2: Model 1+ } \\
\text { marital status, young } \\
\text { children, education }\end{array}$} & \multicolumn{2}{|c|}{$\begin{array}{l}\text { Model 3: Model 1+, } \\
\text { smoking, alcohol and } \\
\text { body mass index }\end{array}$} & \multicolumn{2}{|c|}{$\begin{array}{l}\text { Model 4: Model } 1+ \\
\text { social connections } \\
\text { adjusted for }\end{array}$} & \multicolumn{2}{|c|}{$\begin{array}{c}\text { Model 5: Model 1+ } \\
\text { pain }\end{array}$} & \multicolumn{2}{|c|}{$\begin{array}{l}\text { Model 6: all covariates in } \\
\text { Models } 1-5+\text { any serious } \\
\text { mental or physical health } \\
\text { condition }\end{array}$} \\
\hline & OR & $(95 \% \mathrm{CI})$ & OR & $(95 \% \mathrm{CI})$ & OR & $(95 \% \mathrm{CI})$ & OR & $(95 \% \mathrm{CI})$ & OR & $(95 \% \mathrm{CI})$ & OR & $(95 \% \mathrm{CI})$ \\
\hline \multicolumn{13}{|l|}{ functioning } \\
\hline $6-8$ hours & 1.00 & & 1.00 & & 1.00 & & 1.00 & & 1.00 & & 1.00 & \\
\hline Less than 6 hours sleep & 1.69 & $(1.52-1.87)$ & 1.67 & $(1.50-1.85)$ & 1.57 & $(1.42-1.75)$ & 1.65 & $(1.49-1.83)$ & 1.08 & $(0.96-1.22)$ & 1.05 & $(0.92-1.18)$ \\
\hline More than 8 hours & 1.29 & $(1.17-1.44)$ & 1.27 & $(1.15-1.41)$ & 1.30 & $(1.17-1.44)$ & 1.30 & $(1.17-1.45)$ & 1.29 & $(1.14-1.46)$ & 1.28 & $(1.13-1.45)$ \\
\hline \multicolumn{13}{|l|}{ Poor emotional } \\
\hline \multicolumn{13}{|l|}{ functioning } \\
\hline $6-8$ hours & 1.00 & & 1.00 & & 1.00 & & 1.00 & & 1.00 & & 1.00 & \\
\hline Less than 6 hours sleep & 1.70 & $(1.54-1.88)$ & 1.70 & $(1.54-1.87)$ & 1.60 & $(1.45-1.77)$ & 1.66 & $(1.5-1.83)$ & 1.38 & $(1.24-1.53)$ & 1.30 & $(1.17-1.44)$ \\
\hline More than 8 hours & 1.16 & $(1.05-1.28)$ & 1.12 & $(1.02-1.24)$ & 1.18 & $(1.06-1.30)$ & 1.18 & $(1.07-1.30)$ & 1.14 & $(1.03-1.26)$ & 1.12 & $(1.01-1.24)$ \\
\hline \multicolumn{13}{|l|}{ Poor social functioning } \\
\hline $6-8$ hours & 1.00 & & 1.00 & & 1.00 & & 1.00 & & 1.00 & & 1.00 & \\
\hline Less than 6 hours sleep & 2.02 & $(1.83-2.23)$ & 1.99 & $(1.8-2.2)$ & 1.86 & $(1.69-2.06)$ & 1.96 & $(1.77-2.16)$ & 1.49 & $(1.33-1.66)$ & 1.38 & $(1.23-1.55)$ \\
\hline More than 8 hours & 1.24 & $(1.12-1.38)$ & 1.20 & $(1.08-1.33)$ & 1.25 & $(1.13-1.39)$ & 1.26 & $(1.14-1.40)$ & 1.22 & $(1.09-1.36)$ & 1.20 & $(1.07-1.34)$ \\
\hline
\end{tabular}


Appendix 4. Associations of sleep quality with physical, emotional and social functioning in HILDA wave 13 ( $n=14571$ )

\begin{tabular}{|c|c|c|c|c|c|}
\hline Model 1: age, gender, and & Model 2: Model 1+ marital & Model 3: Model 1+, smoking, & Model 4: Model $1+$ social & Model 5: Model 1+ pain & Model 6: all covariates in Models \\
\hline employment status adjusted & status, young children & alcohol and body mass index & connections adjusted for & & $1-5+$ any serious mental or \\
\hline
\end{tabular}

Sleep quality and duration

\begin{tabular}{|c|c|c|c|c|c|c|c|c|c|c|c|c|}
\hline & OR & $(95 \% \mathrm{CI})$ & OR & $(95 \% \mathrm{CI})$ & OR & $(95 \% \mathrm{CI})$ & OR & $(95 \% \mathrm{CI})$ & OR & $(95 \% \mathrm{CI})$ & OR & $(95 \% \mathrm{CI})$ \\
\hline \multicolumn{13}{|c|}{ Poor physical functioning } \\
\hline No insomnia & 1.00 & & 1.00 & & 1.00 & & 1.00 & & 1.00 & & 1.00 & \\
\hline Insomnia & 2.84 & $(2.58-3.13)$ & 2.82 & $(2.57-3.11)$ & 2.66 & $(2.41-2.93)$ & 2.74 & $(2.49-3.02)$ & 1.57 & $(1.4-1.76)$ & 1.50 & $(1.34-1.68)$ \\
\hline \multicolumn{13}{|c|}{ Poor emotional } \\
\hline \multicolumn{13}{|l|}{ functioning } \\
\hline No insomnia & 1.00 & & 1.00 & & 1.00 & & 1.00 & & 1.00 & & 1.00 & \\
\hline Insomnia & 3.07 & $(2.81-3.36)$ & 3.09 & $(2.82-3.38)$ & 2.90 & $(2.65-3.18)$ & 2.93 & $(2.68-3.20)$ & 2.33 & $(2.12-2.56)$ & 2.13 & $(1.93-2.34)$ \\
\hline \multicolumn{13}{|c|}{ Poor social functioning } \\
\hline No insomnia & 1.00 & & 1.00 & & 1.00 & & 1.00 & & 1.00 & & 1.00 & \\
\hline Insomnia & 3.49 & $(3.19-3.82)$ & 3.48 & $(3.18-3.82)$ & 3.22 & $(2.93-3.53)$ & 3.28 & $(2.99-3.6)$ & 2.30 & $(2.08-2.55)$ & 2.06 & $(1.85-2.29)$ \\
\hline
\end{tabular}

